\title{
RUPTURE OF THE ASCENDING AORTA IN EHLERS-DANLOS SYNDROME AFTER SURGICAL REPAIR OF MULTIPLE ARTERIOVENOUS MALFORMATIONS WITH THE USE OF CARDIOPULMONARY BYPASS
}

\author{
Omke Enno Teebken, MD, ${ }^{\mathrm{a}}$ Matthias Karck, MD, ${ }^{\mathrm{a}}$ Rano Schulz, MD, ${ }^{\mathrm{b}}$ and Axel Haverich, MD, ${ }^{\mathrm{a}}$ Hannover and Kiel, Germany
}

A group of disorders in collagen synthesis with characteristic clinical findings of skin and joint laxity and tissue frailty is known as Ehlers-Danlos syndrome (EDS). Among the 9 different types recognized so far, the most threatening is type IV, which mainly affects the blood vessels and the bowel. Patients have the acute onset of either a hemorrhage from vascular disruption or peritonitis from a ruptured viscus. About 20 different mutations, leading to a deficiency or an altered structure of type III collagen, have been characterized, which explains the existence of various degrees of expression and an autosomal recessive or dominant inheritance. ${ }^{1}$

Clinical summary. A 20-year-old man had a painful swelling and increase in circumference of the left arm. Phlebography revealed thrombosis of the left subclavian vein. During intravenous heparin administration for 3 consecutive days, a massive hematoma developed in the axilla and the pectoralis muscle. The region was surgically exposed and multiple diffuse bleedings had to stopped. Except for a wound dehiscence, the patient recovered well. However, he still had a loud systolic murmur and a palpable thrill above the whole thorax. The mean arterial pressure was $60 \mathrm{~mm} \mathrm{Hg}$ higher at the popliteal artery than at the right brachial artery. In childhood the patient was operated on for pes varus and bilateral inguinal hernias. He had a temporary left hemiparesis after an ischemic insult of the right pons at the age of 16 years. Neither varicose veins nor cardiac signs of EDS nor mitral valve prolapse was seen. The underlying disease remained unclear and further diagnostic investigations were carried out. A computed tomographic scan revealed evidence for an arteriovenous malformation between the brachiocephalic trunk and an enlarged superior vena cava. Magnetic resonance imaging and an intra-arterial angiogram (digital subtraction angiogram) demonstrated an aneurysm of the right subclavian artery, which was $3 \mathrm{~cm}$ in maximum diameter with a postaneurysmal obstruction and a fistula to the superior vena cava (Fig 1).

Surgical technique. After a median sternotomy the right subclavian artery was exposed by means of an infraclavicular

From the Division of Thoracic and Cardiovascular Surgery, Hannover Medical School, ${ }^{a}$ Hannover, and the Department of Pathology, Christian-Albrechts-University, ${ }^{\mathrm{b}}$ Kiel, Germany.

Received for publication March 17, 1998; accepted for publication June 9, 1998

Address for reprints: Omke E. Teebken, MD, Division of Thoracic and Cardiovascular Surgery, Hannover Medical School, CarlNeuberg-Str 1, D-30625 Hannover, Germany.

J Thorac Cardiovasc Surg 1998;116:660-1

Copyright $\odot 1998$ by Mosby, Inc.

0022-5223/98 $\$ 5.00+0 \quad \mathbf{1 2 / 5 4 / 9 2 3 0 7}$

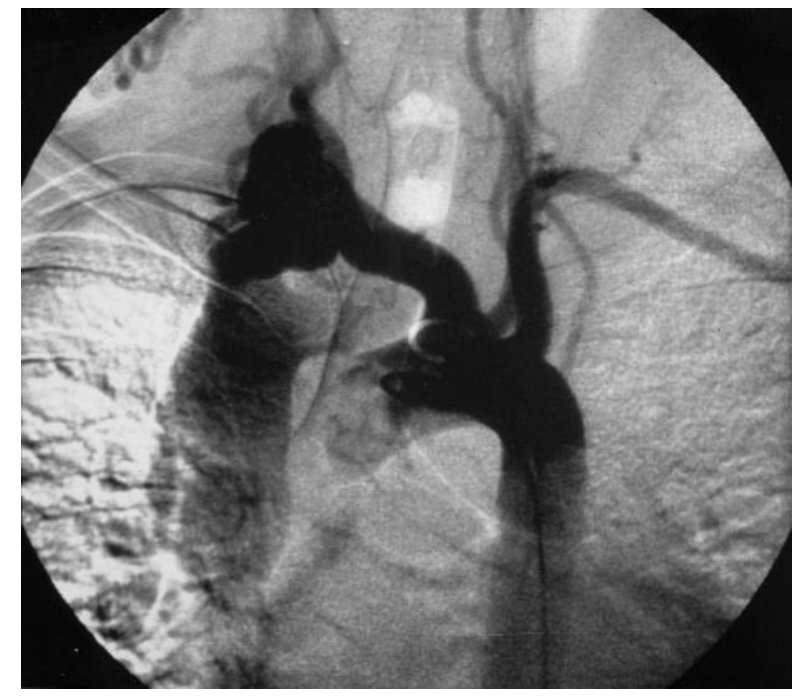

Fig 1. The digital subtraction angiogram of the aortic arch and the supra-aortic vessels shows the connection between the proximal subclavian artery and the enlarged superior vena cava. The subclavian artery is dilated and distally obstructed.

approach. The subclavian artery aneurysm began $1 \mathrm{~cm}$ distally from the brachiocephalic trunk and closely adhered to the dilated right subclavian vein, the vagus, and the recurrent nerve. Many arteriovenous shunts were present. Cardiopulmonary bypass was established via cannulation of the ascending aorta and the right atrium to conserve the patient's blood. The incision of the aneurysm revealed an $8-\mathrm{mm}$ shunt between the superior vena cava and the subclavian artery. After direct closure of the defect and resection of the aneurysm, the brachiocephalic trunk and the subclavian artery were connected with a polytetrafluoroethylene graft (diameter $7 \mathrm{~mm}$, length $9 \mathrm{~cm}$ ) after exclusion of the former multiple arteriovenous shunts.

The early postoperative course was uneventful until the morning of postoperative day 5 , when the patient was found dead in his bed. Postmortem examination revealed a generalized degenerative disease of the great arteries, correlating with stigmata for EDS. The sudden death was caused by rupture of an acute type A dissection of the ascending aorta resulting in cardiac tamponade. Specimens of the excised subclavian aneurysm, the aorta, and other major arteries showed an irregular pattern and arrangement of elastic fibers and a focal decrease of collagen fibers. Furthermore, multiple intima plaques and microcystic mucoid necrosis of the media could 
be demonstrated. Several intimal ruptures were found in the aortic arch and also in the abdominal aorta surrounded by a fresh hematoma in the region of the psoas muscle. The proximal entry of the dissection was located in the ascending aorta, $2.5 \mathrm{~cm}$ distal to the aortic valve.

Comment. Subclavian and axillary artery aneurysms account for about $1 \%$ of all peripheral artery aneurysms. Most of them are caused by an impingement of the artery in thoracic outlet syndrome. Infections, atherosclerosis, trauma, and aneurysms associated with arteritis are rare findings. ${ }^{2}$ Subclavian artery aneurysms in combination with arteriovenous malformations and EDS have not been reported in the past 30 years.

The patient's history of congenital orthopedic defects, the findings at the first operation (left axilla), the secondary wound healing, and the angiograms showing the multiple arteriovenous malformations accompanied by the aneurysmal dilation of the subclavian artery could have led to the suspicion of a hereditary connective tissue disorder like EDS before the second procedure. On the other hand, the patient had no abdominal signs of the disease, and, as confirmed by autopsy, no intestinal abnormalities were present. Correlating to this case, most patients with major vascular complications of EDS have few, if any, of the commonly recognized musculoskeletal and cutaneous abnormalities. ${ }^{3}$ Furthermore, although uncommon, EDS type IV is known as an important potential cause of stroke in early adulthood. Because patients with EDS are prone to complications of spontaneous arterial rupture, noninvasive diagnostic procedures (computed tomography and magnetic resonance imaging, including 3-dimensional image reconstruction) are predominantly applied. Nevertheless, the best visualization of the vascular malformations-necessary for planning the second operation-was achieved with digital subtraction angiography (Fig 1). The bleedings in the left axilla after heparin treatment indicate that anticoagulation therapy may result in increased bruising or bleeding in patients with EDS type IV as well and should be used with caution.

For operative therapy of subclavian artery aneurysms in general, different approaches (eg, sternotomy, posterior left thoracotomy) and special surgical techniques have been described. ${ }^{4}$ Salo and associates ${ }^{5}$ preferred the ligation of the subclavian artery and correction with an extra-anatomic bypass graft between the carotid and subclavian arteries, because this is technically easier and has long-term results similar to those of resection of the aneurysm and direct reconstruction. ${ }^{5}$ With regard to the potential hazardous effects, surgical treatment should consist of the simplest procedure available. In this case, surgical repair of the aneurysm and the fistula between the subclavian artery and the superior vena cava, accompanied by multiple smaller fistulas, carried a risk of significant blood loss. Cardiopulmonary bypass was established to overcome this problem. We are aware that the site of arterial cannulation in the ascending aorta might have been the underlying cause for the subsequent rupture. At autopsy, however, the site of aortic dissection and subsequent rupture was found to be located $2 \mathrm{~cm}$ proximal to the position of the aortic cannula. Bearing in mind the vascular fragility in many of these patients, diagnostic means and potential subsequent surgical therapy should be as minimally invasive and traumatizing as possible.

\section{REFERENCES}

1. Kivirikko KI. Collagens and their abnormalities in a wide spectrum of diseases. Ann Med 1993;25:113.

2. Dougherty MF, Calligaro KD, Savarese RP, De Laurentis DA. Atherosclerotic aneurysms of the intrathoracic subclavian artery: a case report and review of the literature. J Vasc Surg 1995; 21:521-9.

3. Hunter GC, Malone JM, Moore WS, Misiorowski RL, Chvapil M. Vascular manifestations in patients with Ehlers-Danlos syndrome. Arch Surg 1982;117:495-8.

4. Coselli JS, Crawford ES. Surgical treatment of aneurysms of the intrathoracic segment of the subclavian artery. Chest 1987;91: 704-8.

5. Salo JA, Ala-Kulju K, Heikkinen L, Bondestam S, Ketonen P, Luosto R. Diagnosis and treatment of subclavian artery aneurysms. Eur J Vasc Surg 1990;4:271-4. 